\title{
Simultaneous Segmentation, Kinetic Parameter Estimation, and Uncertainty Visualization of Dynamic PET Images
}

\author{
Ahmed Saad ${ }^{1,2}$, Ben Smith ${ }^{1,2}$, Ghassan Hamarneh ${ }^{1}$, and Torsten Möller ${ }^{2}$ \\ ${ }^{1}$ Medical Image Analysis Lab \\ ${ }^{2}$ Graphics, Usability, and Visualization Lab, \\ School of Computing Science, Simon Fraser University, Canada \\ \{aasaad, brsmith, hamarneh, torsten\}@cs.sfu.ca
}

\begin{abstract}
We develop a segmentation technique for dynamic PET incorporating the physiological parameters for different regions via kinetic modeling. We demonstrate the usefulness of our technique on fifteen $\left[{ }^{11} \mathrm{C}\right]$ Raclopride simulated PET images. We show qualitatively and quantitatively that the physiologically based algorithm outperforms two classical segmentation techniques. Further, we derive a formula to compute and visualize the uncertainty encountered during the segmentation.
\end{abstract}

\section{Introduction}

Positron emission tomography (PET) is a functional imaging technique that measures local concentration of a radioactive tracer inside the body. In dynamic PET imaging, a series of 3D images are reconstructed from list-mode data obtained by Gamma coincidence detectors. Kinetic modeling is the process of applying mathematical models to analyze the temporal tracer activity, in order to extract clinically or experimentally relevant information 11. A set of kinetic parameters, resulting from the solution of the inverse problem described by the kinetic model, can describe the tracer behavior in a homogeneous region of a tissue, such as the myocardium, or quantify the densities of the neuroreceptors in the brain. Traditionally, the kinetic modeling process begins by marking a region of interest (ROI) around different functional regions. The PET activity is then averaged over the ROI at each time frame, and a single set of kinetic parameters is estimated by fitting a single kinetic model to the time sequence of average activities.

ROI delineation of functional regions is a tedious, time-consuming, and errorprone task. Further, these delineations may vary depending on the quality of the PET image data and suffer from inter- and intra-operator variability, which may lead to inaccuracies in the estimated average time activity curve (TAC) and the estimated kinetic parameters.

Several approaches have been proposed to identify various functional regions in dynamic PET images. Barber extracted the principal components of a Gamma camera dynamic study followed by factor analysis to identify the fundamental functional changes [2. Di Paola et al. extended Barber's work by applying 
oblique rotation for the loading factors in order to be physiologically relevant [3]. Lin et al. used a Markov random field (MRF) model to differentiate cancerous from normal tissues. They calculated the diagnostic hypoxia fraction and applied spatial constrains to reduce the effect of noise in 2D images [4. Gou et al. utilized the activity histogram of the last frame, or the time-integration of TACs, in order to remove inactive TACs prior to performing hierarchical TAC clustering 5. Liptrot et al. used cluster analysis in order to extract the blood vessel TAC as an alternative to blood sampling [6. Recently, Kamasak et al. simultaneously clustered and estimated each cluster's TAC directly from the projection (sinogram) data, without the need for tomographic reconstruction [7].

All the previous methods consider the PET segmentation and the kinetic parameter estimation as two independent processes, although, in reality, they are very tightly coupled. In other words, the dynamic PET data is first clustered into different homogenous regions and then the kinetic modeling is performed based on the average TAC for each region. Ideally, the segmentation of the dynamic PET data into different functional regions should be based on the physiological processes underlying each region. However, since the physiological processes are captured via the kinetic parameters, and the estimation of kinetic parameters relies on first defining the regions, this creates a dilemma: accurate kinetic parameter estimation requires a segmentation; ideal segmentation requires knowledge of the underlying physiological parameters.

In this paper, we simultaneously segment the PET data and estimate the physiological kinetic parameters in each region. An iterative procedure allows us to deal with the dilemma stated above, where, upon convergence, the segmentation will indeed be based on the underlying kinetic parameters and the kinetic parameters will model the physiological process of each segment. Further, an uncertainty visualization technique is presented to validate the segmentation process.

We first briefly review kinetic parameter modeling (Section 2) and the basic building blocks for clustering TAC data (K-means, MAP-MRF) (Section 33). We then detail our approach for simultaneous PET segmentation and kinetic parameter estimation (Section 4) followed by the uncertainty visualization technique (Section [5). In Section 6, we describe the dataset used in our experiment. We validate our approach and compare it to other standard approaches in Section 7. In Section 8, we conclude with a discussion and future directions.

\section{$2 \quad$ Kinetic Modeling}

The PET tracer kinetics can be described using compartmental models [1]. Each compartment describes a different state of the tracer molecule inside the body. In brain PET imaging, the tracer molecule is injected through the blood and then binds to brain receptors specific to this tracer, or metabolizes inside the tissue. Once tracer paths between compartments are specified, the mass balances between compartments are modelled using a set of ordinary differential equations (ODEs). The ODEs for a corresponding two-tissue compartmental model are described in Figure 1 . 


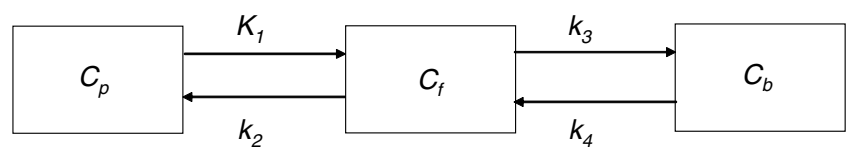

Fig. 1. Two-tissue compartmental model described by 2 ODEs: $d C_{b}(t) / d t=k_{3} C_{f}(t)-$ $k_{4} C_{b}(t) ; d C_{f}(t) / d t=K_{1} C_{p}(t)-k_{2} C_{f}(t)-k_{3} C_{f}(t)+k_{4} C_{b}(t)$

$C_{p}, C_{f}$ and $C_{b}$ are the plasma, intracerebral non-displaceable and specifically bound receptor compartments, respectively. $K_{1}$ is the delivery rate constant $(\mathrm{mL} / \mathrm{min} / \mathrm{g})$, and $k_{2}, k_{3}$ and $k_{4}$ are the first order kinetic rate constants $\left(\mathrm{min}^{-1}\right)$. Since there is no radioactivity prior to scanning, the initial conditions $C_{f}(0)=$ $C_{b}(0)=0$ are used. The estimated kinetic parameters $\left(K_{1}\right.$ and $\left.k_{s}, s \in\{2,3,4\}\right)$ are the solution of the inverse problem capturing the relationship of the output function $\left(C_{f}(t)+C_{b}(t)\right)$ to the input function $\left(C_{p}(t)\right)$ through the unknown parameters [8]. The most common parameter estimation method is least-squares estimation, which seeks the $k_{s}$ that, when inserted in the model's ODEs, produce the best fit to measured data. This can be obtained by minimizing the following objective function:

$$
\sum_{t=1}^{T}\left(\mu(t)-\mu_{f i t}(t)\right)^{2}
$$

where $T$ is the number of time activity measurements at a particular voxel location. $\mu(t)$ is the mean TAC for the functional region under consideration(i.e., mean $C_{f}(t)+C_{b}(t)$ for each functional region). $\mu_{f i t}(t)$ is the model's prediction of tissue activity. $\mu_{f i t}(t)$ can be estimated using an ODE solver based on numerical differentiation formulas [9].

\section{TAC Clustering: K-Means and MAP-MRF}

We base our proposed methods on two basic techniques for segmenting TAC into functional regions: Kd tree-based K-means (KMN) [10] and Maximum a Posteriori MRF [1]. The KMN algorithm iteratively minimizes the following energy function

$$
J_{1}=\sum_{i=1}^{N} \sum_{j=1}^{L}\left\|x_{i}-\mu_{j}\right\|^{2}
$$

where $x_{i}$ is the TAC at each voxel $i$ (e.g. a time-sampled $C_{f}(t)+C_{b}(t)$ ). The time index $t$ is dropped for clarity. $N$ is the number of voxels, $L$ is the number of classes and $\mu_{j}$ is the mean TAC for class $j$. The initial values of $\mu_{j}$ are provided by user-defined seeds into different classes. We use the $L_{2}$ norm as the similarity metric between TACs.

In MRF, beside the image likelihood term in Equation 2, a regularizer for contextual information can be added to discourage assigning different labels to 
neighboring pixels. This can be done in the MRF paradigm by the following energy function:

$$
J_{2}=\sum_{i=1}^{N}\left(\sum_{j=1}^{L}\left\|x_{i}-\mu_{j}\right\|^{2}+\beta \sum_{r=1}^{R} \lambda_{r}\left(x_{i}, x_{r}\right)\right)
$$

where $x_{r}$ is a TAC neighboring $x_{i}$ and $R$ is the size of the neighborhood set. The parameter $\beta$ determines the influence of the regularizer. $\lambda$ is a binary function, returning zero if its arguments belong to the same class and one otherwise.

\section{Simultaneous Segmentation and Estimation of the Kinetic Parameters}

By applying the kinetic modeling process for the means $\mu_{j}$ of all $L$ classes prior to solving Equation 2 or 3. we ensure that the resulting means will be the means capturing the physiological phenomena under consideration, and not the observed TACs from the PET data. To this end, we replace Equations 2 and 3 with two new objective functions described by Equations 4 and 5 , respectively, taking into consideration the kinetic model, as follows.

$$
\begin{gathered}
J_{3}=\sum_{i=1}^{N} \sum_{j=1}^{L}\left\|x_{i}-\mu_{f i t_{j}}\right\|^{2} \\
J_{4}=\sum_{i=1}^{N}\left(\sum_{j=1}^{L}\left\|x_{i}-\mu_{f i t_{j}}\right\|^{2}+\beta \sum_{r=1}^{R} \lambda_{r}\left(x_{i}, x_{r}\right)\right)
\end{gathered}
$$

$\mu_{f i t}$ is the activity TAC produced by solving the kinetic model for each region mean activity $\mu$ using Equation 11. The $k_{s}$, resulting from the solution of the model's ODEs, are the kinetic parameters for each region. The original versions of the KMN and MRF algorithms Equations 2 and 3 are extended to KMN-KM and MRF-KM Equations 4 and 5, where the suffix 'KM' indicates the incorporation of the kinetic model. The algorithm is summarized in Alg, 1

\section{Uncertainty Visualization}

In order to qualitatively evaluate our method, we visualize the uncertainty encountered during the segmentation. We highlight regions where the measured TAC is dissimilar from the kinetic model-based activity. The result of the segmentation technique is assigning each TAC $x_{i}$ to a certain cluster $j$, each of which has a class mean $\mu_{j}$. For each $x_{i}$, we consider $\epsilon_{i j}$ as a measure of uncertainty:

$$
\begin{gathered}
\epsilon_{i j}=\frac{d_{i j}}{1 / L} \\
d_{i j}=\frac{\left\|x_{i}-\mu_{j}\right\|^{2}}{\sum_{l=1}^{L}\left\|x_{i}-\mu_{l}\right\|^{2}}
\end{gathered}
$$




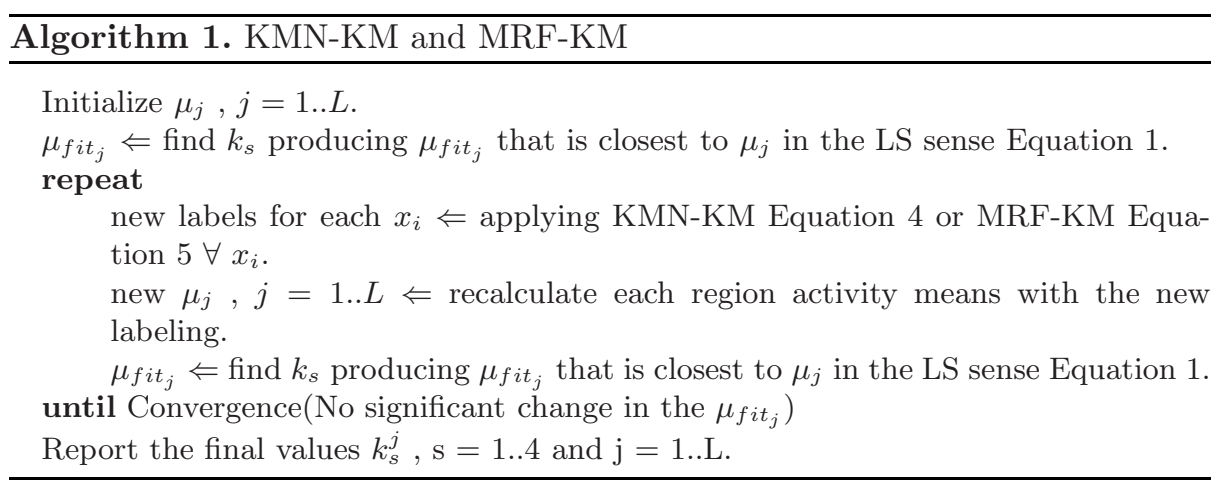

This uncertainty measure $\epsilon_{i j}$ satisfies the two requirements:

- if $x_{i}$ is $100 \%$ certain to belong to class $j$ with mean $\mu_{j}$, then we expect $x_{i}=\mu_{j}$ and therefore $d_{i j}=0$, which gives $\epsilon_{i j}=0$, i.e., no uncertainty, as desired.

- if $x_{i}$ has equal probability to belong to all the clusters we would expect the distance $\left\|x_{i}-\mu_{j}\right\|$ to be equal, independent of the cluster $j$. Therefore $d_{i j}$ $=\frac{1}{L}$, which gives $\epsilon_{i j}=1$, i.e., completely uncertain, as desired.

\section{Materials and Implementation}

We used the publicly available simulated PET dataset PET-SORTEO [12. It provides the ground truth labeling for each TAC. Fifteen $\left[{ }^{11} \mathrm{C}\right]$ Raclopride simulated PET Brain studies accounting for inter-subject anatomical variability have been used. Each data set has dimensions of $128 \times 128 \times 63$ with 26 time steps: 6 with 30s interval, 7 with 60 s interval, 5 with 120 s interval and finally 8 with $300 \mathrm{~s}$ interval with voxel size of $2.11 \times 2.11 \times 2.42 \mathrm{~mm}^{3}$. Each dataset is clustered into 6 different regions (i.e. L = 6): Background BG, Skull SK, Grey matter GM, White matter WM, Cerebellum CM, Putamen PN. We used a two-tissue compartmental model for its suitability for the modeling of $\left[{ }^{11} \mathrm{C}\right]$ Raclopride datasets [13].

The implementation of our algorithms relies on the Insight Toolkit (ITK) 1 . The kinetic modeling process is based on the compartment model kinetic analysis tool (COMKAT) [9].

\section{Results and Discussion}

Figure 2.left shows the input function simulated with PET-SORTEO which represents $C_{p}(t)$ in Figure 1] Figure 2, middle shows the average TAC for each region calculated using the ground truth labeling. Figure 2 right shows the model fitting average TAC for each region after applying the kinetic modeling process to the

\footnotetext{
${ }^{1}$ www.itk.org
} 

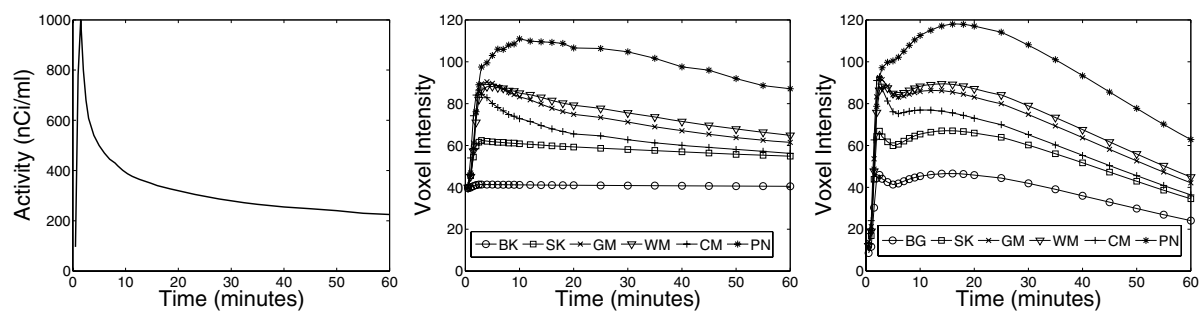

Fig. 2. Left: Input function. Middle: Average TAC for each functional region in the ground truth. Right: Average TAC for each functional region after applying the kinetic modeling process to the ground truth.

ground truth. The difference between Figure 2 middle and Figure 2 right is due to the physiological assumptions about the interaction between the tracer and different tissues in the kinetic modeling process. We used the same initial seeds for each functional region and the same $\beta$ matrix in Equations 3 and 5 when evaluating each of the four methods KMN, MRF, KMN-KM and MRF-KM.

Figure 3 shows qualitatively the comparison between the four algorithms for slice \#40 for patient01. It can be clearly seen that the WM is totally missing in the KMN and MRF algorithms, but KMN-KM and MRF-KM were able to capture it. Further, we see that PN is totally misclassified as WM but it is better captured using the physiological information in KMN-KM and MRF-KM. In order to compare between the algorithms quantitatively for the whole volume, we applied the Dice metric [14 to measure the overlap between different regions as shown in Figure 4. It shows how the KMN-KM and MRF-KM algorithms outperform the classical algorithms KMN and MRF especially in the WM and PN regions. The BG region is excluded from Figure 4 in order to compare between the rest of the active regions, as all the algorithms perform quite well to capture that region as seen in Figure 3 .

It can be seen from the Dice metric that the physiological based algorithms can capture the $\mathrm{PN}$ region, but the value is still low $(\sim 0.21)$. The reason for that can be shown using the uncertainty visualization technique. Figure 5 left shows the result of applying an uncertainty threshold value of $\epsilon=0.5$ to the ground truth labeling after normalizing $\epsilon$ for each region between 0 and 1. Voxels with $\epsilon \leq 0.5$ are selected as "certain" and are rendered in normal colors (as in Figure 3). The remaining "uncertain" voxels are rendered in black. The area around the $\mathrm{PN}$ region in the ground truth image is completely uncertain which explains why it is completely misclassified in the classical algorithms and partially misclassified in the physiologically based algorithms. The uncertainty visualization guides the user to regions of high uncertainty in the segmentation process as shown in Figure 5 right. It shows the uncertainty image of the KNM-KM algorithm with $50 \%$ uncertainty. Doctors are then able to intervene, utilizing their expert knowledge, to provide corrections and iteratively improve and validate the results. The uncertainty metric failed to explain why there is higher confidence in the PN voxels in the main arteries region in our segmentation result 

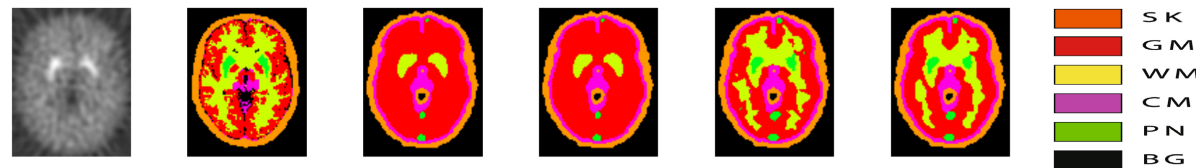

Fig. 3. Comparison between the four algorithms after 10 iterations, from left to right: Slice \# 40 from the axial view of the last time frame of the original PET dataset, ground truth labeling, KMN, MRF, KMN-KM, MRF-KM

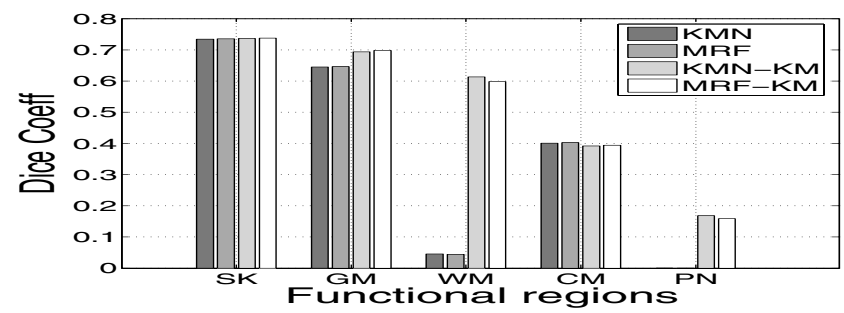

Fig. 4. Performance evaluation between the four algorithms using the Dice metric
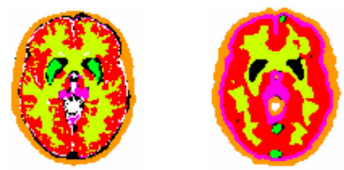

Fig. 5. Uncertainty visualization. Left: Uncertainty image for the ground truth with $50 \%$ uncertainty showing complete uncertainty around the PN. Right: Uncertainty image for the KMN-KM algorithm with 50\% uncertainty. The background voxels are colored in white to emphasize the uncertainty values in black.

in Figure 5.right. It resulted from the fact that the $\mathrm{PN}$ and main arteries have very similar activities based on the TAC $L_{2}$ distance used.

\section{Conclusion and Future Work}

In this paper, we showed qualitatively and quantitatively that incorporating the physiological model that describes the kinetics of the radioactive tracer into the segmentation techniques produces better results over the classical segmentation techniques. We also showed how we can visualize the uncertainty encountered during the segmentation process. This provides an efficient way to incorporate the user interaction and validate the segmentation results.

Our algorithm depends on the presence of the input function which describes the amount of tracer into the plasma to fully solve the kinetic modeling process. In clinical settings, invasive methods of extracting the input function are the golden standard [1]. We plan to include the non-invasive kinetic models into 
the segmentation technique. Further, we need to investigate the performance of our algorithm with real datasets with different noise levels. Our uncertainty visualization technique needs to be incorporated into an efficient user interaction model for editing the segmentation results instead of the standard relabeling on a per-voxel basis.

Acknowledgements. This work has been supported in part by NSERC.

\section{References}

1. Morris, E.D., Endres, C.J., Schmidt, K.C., Christian, B.T.: Kinetic modeling in Positron Emission Tomography. In: Wernick, M., Aarsvold, J.N. (eds.) Emission Tomography: The Fundamentals of PET and SPECT, Academic, San Diego (2004)

2. Barber, D.C.: The use of principal components in the quantitative analysis of gamma camera dynamic studies. Phys. Med. Biol. 25, 283-292 (1980)

3. Di Paola, R., Bazin, J.P., Aubry, F., Aurengo, A., Cavailloles, F., Herry, J.Y., Kahn, E.: Handling of dynamic sequences in nuclear medicine. IEEE Trans. Nucl. Sci. NS29, 1310-1321 (1982)

4. Lin, K.P., Lou, S.L., Yu, C.L., Chung, B.T., Wu, L.C., Liu, R.S.: Markov random field method for dynamic PET image segmentation. In: Proceedings of the SPIE Image Processing, pp. 1198-1204 (1998)

5. Guo, H., Renaut, R., Chen, K., Reiman, E.: Clustering huge data sets for parametric PET imaging. Journal of Biosystems 71, 81-92 (2001)

6. Liptrot, M., Adams, K., Martiny, L., Pinborg, L., Lonsdale, M., Olsen, N., Holm, S., Svarer, C., Knudsen, G.M.: Cluster analysis in kinetic modelling of the brain: a noninvasive alternative to arterial sampling. NeuroImage 21(2), 483-493 (2004)

7. Kamasak, M.E., Bayraktarb, B.: Unsupervised clustering of dynamic PET images on the projection domain. In: Proceedings of the SPIE Medical Imaging, pp. 1539$1548(2006)$

8. Carson, R.E.: Tracer kinetic modeling in PET. In: Bailey, D.L., Townsend, D.W., Walk, P.E., Maisey, M.N. (eds.) Positron Emission Tomography, Basic Sciences, Springer, Heidelberg (2005)

9. Muzic, R., Cornelius, S.: COMKAT: compartment model kinetic analysis tool. J. Nucl. Med. 42(4), 636-645 (2001)

10. Kanungo, T., Mount, D.M., Netanyahu, N.S., Piatko, C.D., Silverman, R., Wu, A.Y.: Efficient k-means clustering algorithm: Analysis and implementation. IEEE PAMI 24, 881-892 (2002)

11. Besag, J.: On the statistical analysis of dirty pictures. Journal of Royal Statistics 48, 259-302 (1986)

12. Reilhac, A., Batan, G., Michel, C., Grova, C., Tohka, J., Costes, N., Evans, A.C.: Validation of PET SORTEO: a platform for simulating realistic PET studies and development of a database of simulated PET volumes. IEEE Trans. Nucl. Sci. 52, 1321-1328 (2004)

13. Lammertsma, A., Bench, C., Hume, S., Osman, S., Gunn, K., Brooks, D., Frackowiak, R.: Comparison of methods for analysis of clinical [11C]raclopride studies. J. Cereb. Blood Flow Metab. 16, 42-52 (1996)

14. Dice, L.R.: Measures of the amount of ecologic association between species. Ecology 26, 297-302 (1945) 